\title{
RELAÇÃO DOSE-DEPENDENTE DO USO CRÔNICO DE FENITOÍNA E ATROFIA CEREBELAR EM PACIENTES COM EPILEPSIA
}

\author{
ANDRÉDEL NEGRO*, CLARISSA DEROSALMEIDA DANTAS ${ }^{*}$, VERÔNICA ZANARDI***, \\ MARIA AUGUSTA MONTENEGRO ** FERNANDO CENDES ${ }^{* * *}$
}

\begin{abstract}
RESUMO - O uso crônico da fenitoína ou intoxicação aguda por essa droga produzem lesão cerebelar permanente com atrofia do vermis e hemisférios cerebelares, que pode ser evidenciada através de exames de neuroimagem. O objetivo deste estudo foi avaliar a correlação entre a dosagem e o tempo de uso da fenitoína com a ocorrência de atrofia cerebelar. Foram realizados levantamento de prontuários para a obtenção de dados clínicos e análise de tomografias de crânio para avaliação de atrofia cerebelar. Dos 66 pacientes estudados, 18 apresentaram atrofia moderada a severa, 15 atrofia leve e 33 foram considerados normais. Os pacientes com atrofia cerebelar moderada a severa foram aqueles com maior exposição à fenitoína (uso prolongado e dose total), apresentando diferença estatisticamente significativa se comparados aos pacientes com atrofia leve ou sem atrofia ( $\mathrm{p}=0.02)$. Além disso, no subgrupo de pacientes em uso de fenitoína, aqueles com atrofia moderada a severa possuíam níveis séricos de fenitoína significativamente mais elevados que os pacientes com atrofia leve ou sem atrofia $(\mathrm{p}=0.008)$. Não houve relação entre duração do tratamento e dose de outros anticonvulsivantes e presença e grau de atrofia cerebelar. Os pacientes mais velhos apresentaram maior grau de atrofia cerebelar, indicando que o fator idade ou tempo de epilepsia, ou ambos, pode ser importante na determinação de degeneração cerebelar. Concluímos que apesar da possibilidade de lesão cerebelar relacionada a crises epilépticas repetidas, a contribuição da fenitoína pode ser claramente estabelecida como um dos determinantes da atrofia cerebelar, sobretudo naqueles pacientes com altas doses por tempo prolongado e níveis séricos elevados.
\end{abstract}

PALAVRAS-CHAVE: fenitoína, atrofia cerebelar, epilepsia.

\section{Dose-related cerebellar atrophy in patients with epilepsy using phenytoin}

ABSTRACT - The chronic treatment with phenytoin or the acute intoxication by this drug may cause permanent cerebellar injury with atrophy of cerebellum vermis and hemispheres, which can be detected by neuroimaging studies. The aim of the present study was to investigate the correlation between the dosage and duration of treatment with phenytoin and the occurrence of cerebellar atrophy. Sixty-six patients were studied and had their tomographies analyzed for cerebellar atrophy. Of the 66 patients studied, 18 had moderate/severe atrophy, 15 had mild atrophy and 33 were considered to be normal. The patients with moderate/severe atrophy were those with higher exposure to phenytoin (longer duration of treatment and higher total dosage) showing statistically significant difference when compared to patients with mild atrophy or without atrophy $(\mathrm{p}=0.02)$. Further, the patients with moderate/severe atrophy had serum levels of phenytoin statistically higher than those of patients with mild atrophy or without atrophy $(\mathrm{p}=0.008)$. There was no association between other antiepileptic drugs dosage or duration of treatment and degree of cerebellar atrophy. We also found that older patients had cerebellar atrophy more frequently, indicating that age or duration of the seizure disorder may also be important in the determination of cerebellar degeneration in these patients. We conclude that although there is a possibility that repeated seizures contribute to cerebellar damage, long term exposure to phenytoin, particularly in high doses and toxic serum levels, cause cerebellar atrophy.

KEY WORDS : phenytoin, cerebellar atrophy, epilepsy, seizures.

Departamento de Neurologia e Departamento de Radiologia de Faculdade de Ciências Médicas da Universidade Estadual de Campinas (FCM/UNICAMP): *Acadêmico de Medicina, **Médica Residente, ****Professor Assistente Doutor. Apoio: FAPESP, Bolsa de iniciação científica. Aceite: 6-janeiro-2000.

Dr. Fernando Cendes - Departamento de Neurologia - FCM / UNICAMP - Caixa Postal 6111 - 13081-970

Campinas SP - Brasil. FAX 19289 0880. Email: fcendes@unicamp.br 
A difenil-hidantoína (DPH) é droga de primeira linha para tratamento de muitas formas de epilepsia, tanto pela sua boa eficácia quanto pelo seu custo acessível. Seu mecanismo de ação consiste no efeito estabilizador da membrana neuronal através da inibição dos canais de sódio sensíveis à voltagem, reduzindo o fluxo iônico tanto durante o repouso quanto durante o potencial de ação ${ }^{1}$. Dessa maneira, a DPH impede que haja deflagrações neuronais repetitivas provocadas pela passagem de corrente intracelular sem causar depressão geral do sistema nervoso central (SNC). Seu uso, entretanto, não é isento de efeitos adversos, sendo o mais comum a disfunção cerebelar após intoxicação aguda, levando a sintomas como diplopia e ataxia. Atrofia cerebelar irreversível pode ocorrer após intoxicação aguda $^{2}$ e também após o uso crônico da fenitoína ${ }^{3-7}$. Estudos anteriores indicam que a atrofia cerebelar devido ao efeito tóxico direto da droga sobre as células de Purkinje ou pela hipóxia causada pelas crises generalizadas tônico-clônicas $^{3-7}$, ou por esses dois fatores atuando sinergicamente ${ }^{8-11}$.

O objetivo deste estudo foi correlacionar a atrofia cerebelar com o uso crônico da DPH.

\section{MÉTODO}

Realizamos estudo retrospectivo através do levantamento de prontuários de 127 pacientes acompanhados no Ambulatório de Epilepsia de Serviço do Neurologia do Hospital das Clínicas-UNICAMP. Dentre os prontuários, selecionamos aqueles que: apresentavam dados completos quanto a sexo e idade dos pacientes; tipo de droga anti-epiléptica (DAE) em uso e dose atual; tempo de uso da DAE; história pregressa de quadro clínico sugestivo de intoxicação medicamentosa pela droga (ataxia, diplopia, vertigem e nistagmo, entre outras) verificando, sempre que possível, o nível sérico da DAE. Por fim, verificamos se o paciente apresentava alguma doença de base, como neurocisticercose, trauma crânio-encefálico e calcificações cerebrais, que pudesse estar associada à atrofia cerebelar.

Após coletados os dados, foram excluídos 59 pacientes cujos prontuários não preencheram esses quesitos e 2 pacientes foram excluídos por apresentarem sequela de lesão isquêmica na tomografia computadorizada (TC) dificultando a determinação dos possíveis fatores causais de atrofia. Avaliamos o grau de atrofia cerebelar em 66 pacientes através da TC de crânio, de forma "cega" em relação às informações clínicas. A avaliação foi conduzida, em separado, por dois profissionais com experiência em TC, sendo um radiologista e outro neurologista clínico, ambos sem informação sobre a DAE em uso e tempo de exposição à droga do paciente avaliado.

Para tornarmos o estudo mais fidedigno, randomizamos a ordem dos usuários de DPH e os usuários de outras DAE. Além disso, introduzimos no estudo radiológico $10 \mathrm{TC}$, consideradas normais previamente, de pacientes que não apresentavam história de crises convulsivas ou uso de DAE e que realizaram TC para investigação de cefaléia.

A avaliação radiológica da atrofia cerebelar nas TCs de crânio seguiu os parâmetros de Koller e col. ${ }^{12}$ com pequenas modificações:

1) Vermis cerebelar - atrofia leve quando se notam dois ou três sulcos na linha média (Fig 1); atrofia moderada a severa quando mais de três sulcos são evidenciados em associação com redução de volume significativa de mais de um dos segmentos do vérmis (Fig 2 e 3).

2) Hemisférios cerebelares - atrofia leve quando as folhas do cerebelo são visíveis, isto é, quando é possível observar sulcos proeminentes entre elas, porém sem dilatação importante das cisternas da fossa posterior (Fig 1); atrofia moderada a severa quando for observada uma redução de volume significativa de todo o cerebelo com conseqüente aumento das cisternas da fossa posterior (Figs 2 e 3 ).

Foram investigadas 66 TCs de crânio de pacientes com epilepsia, sendo 37 de usuários de fenitoína e 29 em uso de outras DAE que não utilizaram fenitoína, além de 10 de pacientes acompanhados no ambulatório de cefaléia como grupo controle, conforme mencionado anteriormente.

Após avaliação das TCs, classificamos as atrofias segundo os critérios de $\mathrm{Koller}^{12} \mathrm{em}$ : ausente ou leve, e moderada a grave, combinando a avaliação do vermis e dos hemisférios cerebelares. Inicialmente, avaliamos também o tamanho do quarto ventrículo, porém descartamos esse dado devido à grande variação nos resultados.

Depois dessa avaliação radiológica, empregamos o Drug Score proposto por Kaneko e col. ${ }^{13}$. Este Drug Score possibilita a comparação entre as doses das diferentes DAEs. Calculamos então a quantidade total das DAEs usadas por cada paciente durante todo o tempo de tratamento até a realização da TC. de pacientes.

Após tabulação dos dados, aplicamos o teste de Fisher para avaliar diferenças estatísticas entre os grupos 


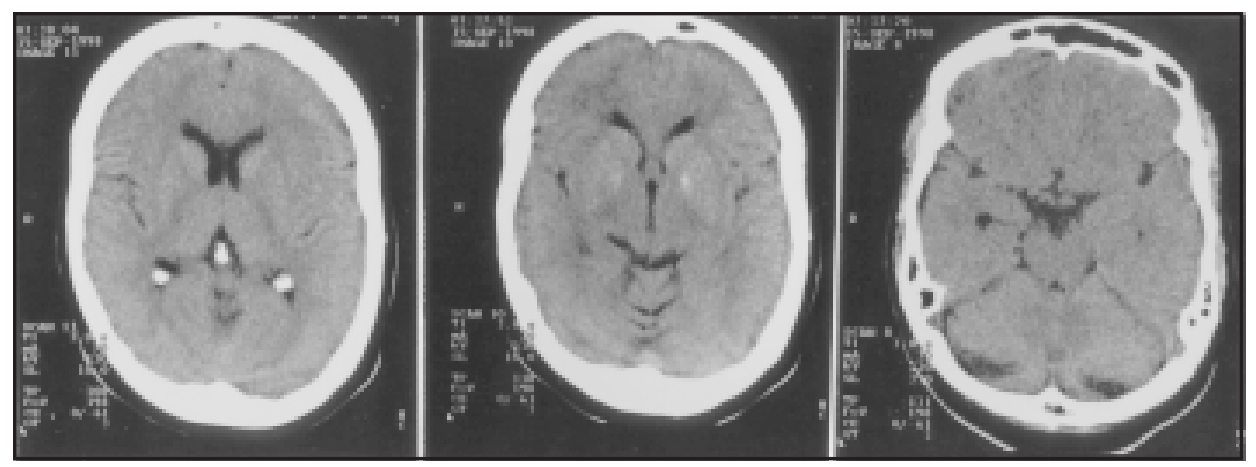

Fig 1. Tomografia computadorizada considerada como atrofia cerebelar leve de predomínio no vermis.

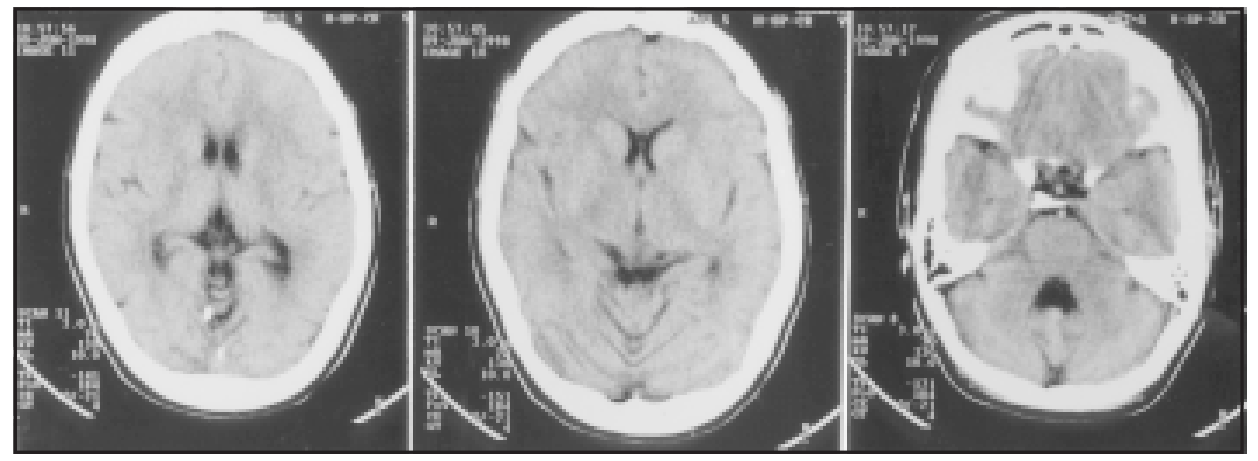

Fig 1. Tomografia computadorizada considerada como atrofia cerebelar leve de predomínio no vermis.

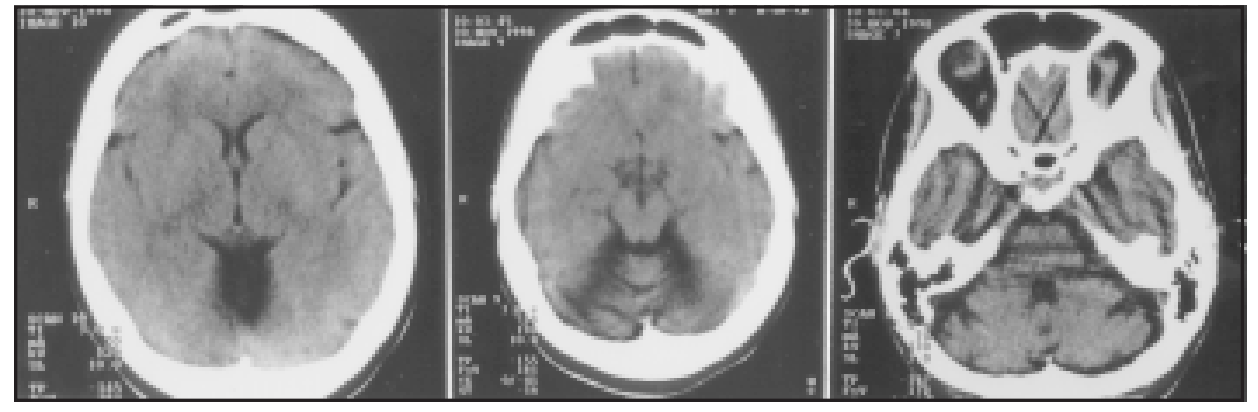

Fig 3. Tomografia computadorizada considerada como atrofia cerebelar severa.

\section{RESULTADOS}

Dos 66 pacientes com epilepsia avaliados, 37 utilizavam fenitoína e 29 outras DAE. Ao compararmos o grupo de pacientes em uso de fenitoína versus o grupo de pacientes em uso de outros anticonvulsivantes, verificamos que os usuários de fenitoína apresentaram sintomas de origem vestibulocerebelar $(\mathrm{p}=0,04)$ e níveis séricos tóxicos $(\mathrm{p}=0,0004)$ em maior freqüência (Tabela 1$)$. A duração e severidade da epilepsia foi semelhante nos dois grupos. Não houve registro de estado de mal epiléptico nos pacientes estudados. 
Tabela 1. Comparação entre os grupos de pacientes usuários de fenitoína e de outras drogas antiepilépticas.

\begin{tabular}{lccc}
\hline & Fenitoína & Outras drogas & p* \\
\hline Gênero (masc/fem) & $17 \mathrm{M} / 21 \mathrm{~F}$ & $9 \mathrm{M} / 22 \mathrm{~F}$ & $\mathrm{Ns}$ \\
Idade (anos) & $38,5 \pm 2,3$ & $38,9 \pm 2,8$ & $\mathrm{Ns}$ \\
Duração tratamento (meses) & $79,6 \pm 9,8$ & $77,9 \pm 9,04$ & $\mathrm{Ns}$ \\
Síndrome cerebelar (sim/não) & $5 \mathrm{~S} / 33 \mathrm{~N}$ & $31 \mathrm{~N}$ & 0,04 \\
Intoxicação (sim/não) & $8 \mathrm{~S} / 30 \mathrm{~N}$ & $31 \mathrm{~N}$ & 0,0004 \\
\hline
\end{tabular}

Resultados expressos com média \pm erro padrão.

* teste de Fisher. Ns, diferença estatística não significativa

Uma análise individual mostrou que apenas 4 pacientes fizeram uso regular de DPH por mais de 10 anos (média em anos: 13,63 \pm 1,30), todos apresentando atrofia cerebelar importante. Já entre usuários de outras DAE, 6 fizeram uso regular por mais de 10 anos $(11,31 \pm 0,87)$, sendo que apenas 1 apresentou atrofia importante.

Entre os usuários de fenitoína, 13 apresentaram atrofia moderada a severa, 24 atrofia leve ou ausência de atrofia. Os pacientes com atrofia cerebelar moderada a severa apresentaram maior exposição à fenitoína comparados aos pacientes com atrofia leve ou sem atrofia. Houve diferença significativa quanto ao tempo de uso $(\mathrm{p}=0,03)$ e dose total utilizada $(\mathrm{p}=0,02)$. Além disso, os pacientes com atrofia moderada a severa possuíam níveis séricos de fenitoína significativamente mais elevados que os de pacientes com atrofia leve ou sem atrofia $(\mathrm{p}=0,008)$ (Tabela 2).

Entre os usuários de outras DAE, 5 apresentaram atrofia moderada a severa e 24 atrofia leve ou ausência de atrofia. Não houve relação entre duração de tratamento e dose dos outros anticonvulsivantes e grau de atrofia cerebelar (Tabela 3). Observamos porém, uma diferença estatística entre idade média dos indivíduos com atrofia moderada a severa e aqueles com atrofia leve ou sem atrofia $(\mathrm{p}=0,008)$; indicando que o fator idade e/ou duração da epilepsia também deve(m) ser importante na determinação da atrofia cerebelar nestes pacientes.

Tabela 2. Comparação entre os 37 pacientes em uso de fenitoína de acordo com atrofia cerebelar.

\begin{tabular}{lccc}
\hline & Atrofia moderada a severa & Atrofia leve ou ausente & p* \\
\hline Gênero (masc/fem) & $4 \mathrm{M} / 9 \mathrm{~F}$ & $10 \mathrm{M} / 14 \mathrm{~F}$ & $\mathrm{Ns}$ \\
Idade (anos) & $39,3 \pm 4,0$ & $37,9 \pm 3,0$ & $\mathrm{Ns}$ \\
Nível Sérico (mg/dl) & $33,42 \pm 5,2$ & $16,62 \pm 3,2$ & 0,008 \\
Duração tratamento (meses) & $109,4 \pm 23,2$ & $63,2 \pm 7,5$ & 0,03 \\
Dose (mg) & $140,4 \pm 53,5$ & $368,7 \pm 141,4$ & $\mathrm{Ns}$ \\
Dose total (g) & $898,0 \pm 178,6$ & $535,1 \pm 61,2$ & 0,02 \\
Síndrome cerebelar (Sim/Não) & $2 \mathrm{~S} / 11 \mathrm{~N}$ & $3 \mathrm{~S} / 21 \mathrm{~N}$ & $\mathrm{Ns}$ \\
Intoxicação (Sim/Não) & $8 \mathrm{~S} / 5 \mathrm{~N}$ & $5 \mathrm{~S} / 19 \mathrm{~N}$ & 0,004 \\
\hline
\end{tabular}

Resultados expressos com média \pm erro padrão.

* teste de Fisher. Ns, diferença estatística não significativa 
Tabela 3. Comparação entre os 29 pacientes usuários de outras DAE de acordo com atrofia cerebelar.

\begin{tabular}{lccc}
\hline & Atrofia moderada a severa & Atrofia leve ou ausente & p* \\
\hline Gênero (masc/fem) & $3 \mathrm{M} / 2 \mathrm{~F}$ & $6 \mathrm{M} / 18 \mathrm{~F}$ & $\mathrm{Ns}$ \\
Idade (anos) & $55,2 \pm 7,1$ & $35,4 \pm 2,8$ & 0,008 \\
Duração tratamento (meses) & $64,8 \pm 27,7$ & $81,8 \pm 10,2$ & $\mathrm{Ns}$ \\
Dose (drug score) & $22350,94 \pm 23884,66$ & $20579,50 \pm 23459,84$ & $\mathrm{Ns}$ \\
Dose total (drug score) & $18738,0 \pm 11274,5$ & $22984,6 \pm 4870,5$ & 0,7187 \\
Síndrome cerebelar (sim/não) & $5 \mathrm{~N}$ & $28 \mathrm{~N}$ & $\mathrm{Ns}$ \\
Intoxicação (sim/não) & $5 \mathrm{~N}$ & $28 \mathrm{~N}$ & $\mathrm{Ns}$ \\
\hline
\end{tabular}

Resultados expressos com média \pm erro padrão médio.

* teste de Fisher. Ns= diferença estatística não significativa

\section{DISCUSSÃO}

Embora haja relatos na literatura ${ }^{1-10}$ de ataxia permanente associada ao uso de fenitoína em altas doses e por tempo prolongado, existem poucos estudos com casuística suficiente para definir a etiologia da atrofia cerebelar. O estabelecimento de correlação inequívoca entre o uso de fenitoína e a atrofia cerebelar é difícil pois, na maioria dos casos, além do uso da medicação anticonvulsivante, os pacientes também apresentam crises tônico-clônicas generalizadas e hipóxia decorrente destas crises. Alterações cerebelares caracterizadas por lesão das células de Purkinje foram demonstradas experimentalmente em animais com níveis séricos de fenitoína elevados ${ }^{14,15}$. Rapport \& Shaw ${ }^{4}$ estabeleceram com maior segurança a relação entre fenitoína e atrofia cerebelar ao relatar este achado em um paciente tratado profilaticamente com fenitoína e que nunca havia apresentado crise epiléptica. Entretanto, colaboram para a controvérsia dos estudos ${ }^{3,8}$ que demonstram ser a hipóxia, devida às crises generalizadas frequentes, uma provável causa de lesão cerebelar.

Os resultados do nosso estudo estão de acordo com a literatura ${ }^{3,7,9}$, e mostram que pacientes em uso crônico de fenitoína, principalmente aqueles com dosagem sérica elevada e com maior tempo de tratamento, apresentam atrofia cerebelar mais severa se comparados aos pacientes tomando fenitoína em doses menores e também aos pacientes em uso de outras DAEs, independente da dose e do tempo de tratamento.

É interessante observar que os pacientes mais velhos apresentaram maior grau de atrofia cerebelar, indicando que o fator idade e/ou tempo de epilepsia pode(m) ser importante(s) na determinação de degeneração cerebelar.

Concluímos que apesar de haver a possibilidade de degeneração secundária à hipóxia causada por crises epilépticas repetidas, a contribuição da fenitoína pode ser claramente estabelecida como um dos determinantes da atrofia cerebelar, sobretudo naqueles pacientes com altas doses por tempo prolongado e níveis séricos elevados.

\section{REFERÊNCIAS}

1. Bruni J. Phenytoin toxicity. In Levy RH Meldrum BS (eds). Antiepileptic drugs. Ed.4, New York: Raven Press, 1995:345-350

2. Kuruvilla T, Bharucha NE. Cerebellar atrophy after acute phenytoin intoxication. Epilepsia 1997; 38: 500-502.

3. Botez M I, Attig E, Vezina JL. Cerebellar atrophy in epileptic patients. Can J Neurol Sci 1988;15:299-303.

4. Rapport RL, Shaw CM. Phenytoin-related cerebellar degeneration without seizures. Ann Neurol 1977;2:437-439.

5. Selhorst JB, Kaufman B, Horowitz SJ. Diphenylhydantoin-induced cerebellar degeneration. Arch Neurol 1972;27:453-455

6. Gathak NR, Santoso RA, McKinney WM. Cerebellar degeneration following long-term phenytoin therapy. Neurology $1976 ; 26: 818-820$. 
7. McLain LW, Martain JT, Allen JH. Cerebellar degeneration due to chronic phenytoin therapy. Ann Neurol 1980;7:18-23.

8. Dam M. Number of Purkinje cells in patients with grand mal epilepsy treated with diphenylhydantoin. Epilepsia 1970;11:375-386

9. Ney GC, Lantos G, Barr WB, Schaul N. Cerebellar atrophy in patientes with long-term phenytoin exposure and epilepsy. Arch Neurol 1994;51:767-771.

10. Luef G, Chemelli A, Birbamer G, Aichnar F, Bauer G. Phenytoin overdosage and cerebellar atrophy in epileptic patients: clinical and MRI findings. Eur Neurol 1994;(Suppl 1):79-81.

11. Luef G, Burtscher J, Kremser C, et al. Magnetic resonance volumetry of the cerebellum in epileptic patients after phenytoin overdosages. Eur Neurol 1996;36:273-277.

12. Koller WC, Glatt SL, Perlik S, Huckman MS, Fox JH. Cerebellar atrophy demonstrated by computed tomography. Neurology 1981;31:405-412

13. Kaneko S, Otani K, Fukushima Y, et al. Teratogenicity of antiepileptic drugs: analysis of possible risk factors. Epilepsia $1988,29,459-467$

14. Kiefer R, Knoth R, Anagnostopoulos J, Volk B. Cerebellar injury due to phenytoin: identification and evolution of Purkinje cell axonal swellings in deep cerebellar nuclei of mice. Acta Neuropathol (Berl) 1989;77:289-298.

15. Volk B, Kirchgassner N. Damage of Purkinje cell axons following chronic phenytoin administration: an animal model of distal axonopathy. Acta Neuropathol (Berl) 1985;67:67-74. 